\title{
The study on Design and Algorithm of Robot Control System
}

\author{
Jin Yao \\ School Department of mechanics and materials engineering, Wuzhou University, Wuzhou 543000, \\ China
}

Keywords: Self-balancing robot; Data Fusion; Adaptive Fuzzy Control.

\begin{abstract}
The self-balancing robot sensor characteristics, proposed indirect Kalman filter algorithm based on a gyroscope and accelerometer data fusion, optimal inclination and angular velocity estimates. The steering system is decoupled into two subsystems forward. For forward subsystem has strong nonlinear, demonstrated the feasibility of the application of nonlinear systems based on approximate linearization, exact linearization and intelligent control of the control strategy.
\end{abstract}

\section{Introduction}

And the inverted pendulum systems, self-balancing robot not only required maintaining a balance, you must also achieve the desired movement. The most common way is to allow the movement of the robot at a given forward speed, if the need to do more complex movement (e.g., track the desired trajectory) can be given by the controller to achieve a speed corresponding to the instruction sequence. Due to the special nature of the structure, must make the robot speed servo controller on the basis of maintaining homeostasis, which is the difficulty of the subject. Because the goal of self-balancing robot applications is very broad, it may encounter unexpected complex environments, such as the road may not be smooth, there is a collision or interference by the wind.

\section{Decoupling structure determination and control system}

Applied to the left and right wheel motor control voltage, drive around the wheel movement, the robot will be able to achieve progress and steering. The forward speed of the robot speed is half the sum of two rounds, and the steering speed is directly proportional to the speed difference between the two rounds. Balance the subsystem to the forward advance speed and the vehicle body inclination angular velocity of the state vector, two half the sum of the motor voltage is input, the system can be obtained by the equation (1) is described.

$$
\left[\begin{array}{l}
\bar{x}_{1} \\
\bar{x}_{2} \\
\bar{x}_{3}
\end{array}\right]=\left[\begin{array}{l}
-\frac{\left(a_{1}+a_{2} \cos x_{2}\right)}{1-d_{1} \cos ^{2} x_{2}} x_{1}-\frac{a_{3} \sin 2 x_{2}}{1-d_{1} \cos ^{2} x_{2}}+\frac{a_{4} \sin x_{2}}{1-d_{1} \cos ^{2} x_{2}} x_{3}^{2} \\
x_{3} \\
-\frac{\left(b_{1}+b_{2} \cos x_{2}\right)}{1-d_{1} \cos ^{2} x_{2}} x_{1}-\frac{b_{3} \sin 2 x_{2}}{1-d_{1} \cos ^{2} x_{2}}+\frac{b_{4} \sin x_{2}}{1-d_{1} \cos ^{2} x_{2}} x_{3}^{2}
\end{array}\right]+\left[\begin{array}{c}
\frac{\left(a_{5}+a_{6} \cos x_{2}\right)}{1-d_{1} \cos ^{2} x_{2}} \\
0 \\
\frac{\left(b_{5}+b_{6} \cos x_{2}\right)}{1-d_{1} \cos ^{2} x_{2}}
\end{array}\right] u
$$

The steering angle of the subsystem to the state vector of the angular velocity $\omega$, the voltage difference between the two motors half of input, the system can (2) described by the formula.

$$
\bar{x}_{4}=-c_{1} \bar{x}_{4}+c_{2} \bar{u}
$$

After decoupling, respectively, can be designed for each of the two sub-controllers, the controller will calculate the balance of the forward advance according to a task $\mathrm{u}$, the steering controller will be solved according to the steering requirements of the conjugate value of $u$, and finally by the following formula the control voltage then obtained about the motor.

\section{Control Simulation}

According to fuzzy adaptive algorithm design, simulation results are given under this algorithm. Figure 1 shows the case when the robot is in the initial state [ $\left.\begin{array}{lll}0 & 1.5 & 0\end{array}\right]$ to return it to the controller is still successful balance, an excellent balance of properties. Figure 2 is a reference input is $r=1$ in response to the forward speed of the robot, the robot tracking precision, fast response. 


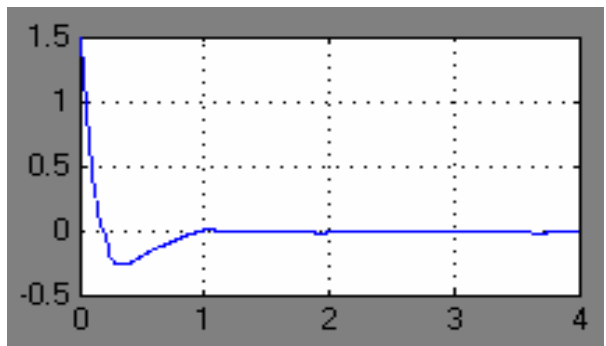

Fig.1 Balancing control under initial state [0 1.50$]$

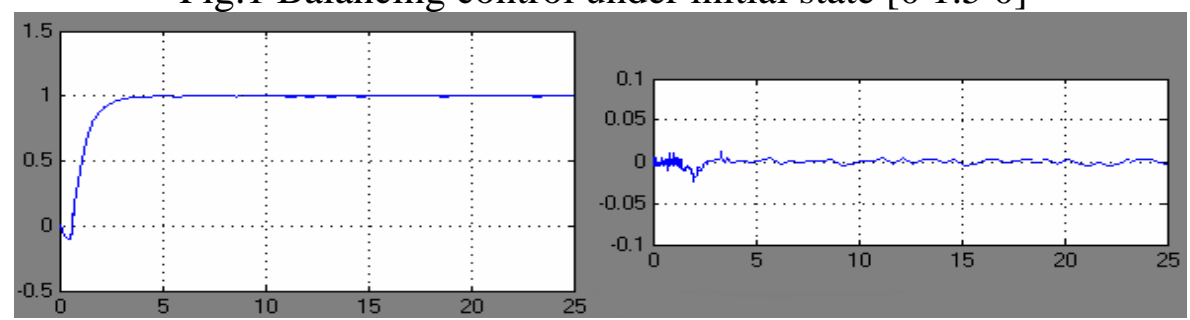

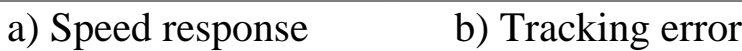

Fig. 2 Responses for FMRLC (step input sequence)

When the reference input is a sinusoidal signal, Figure 3 shows the output of the reference model, and Figure 4 for the speed of the output response curve ${ }^{[1-4]}$. The simulation results show the effectiveness FMRLC, the controller has finished the control tasks. In order to verify FMRLC adaptive function, in other parameters unchanged, so the system by $\mathrm{w}=\left[\begin{array}{lll}0.2 & 0.1\end{array}\right] \mathrm{T}$ of disturbance, the robot body mass becomes $3 \mathrm{~kg}$.

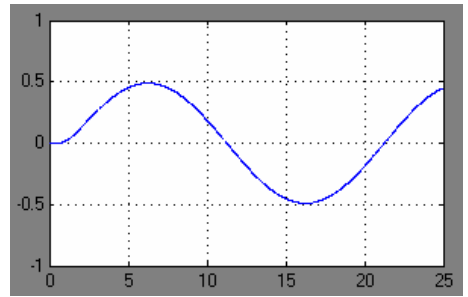

Fig.3 Reference model output (sinusoidal input sequence)

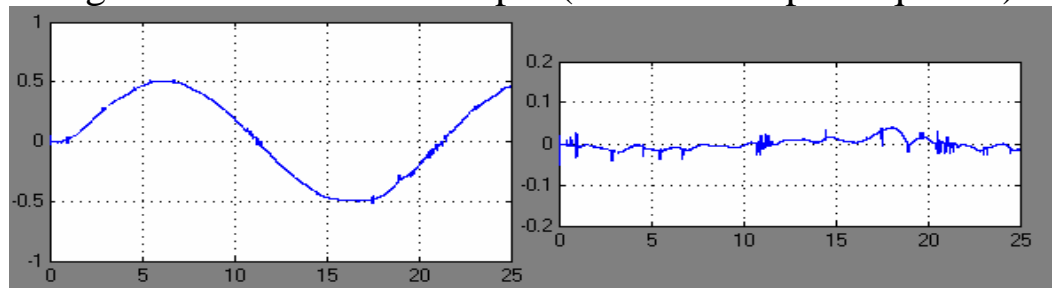

a) Speed response

b) Tracking error

Fig.4 Responses for FMRLC (sinusoidal input sequence)

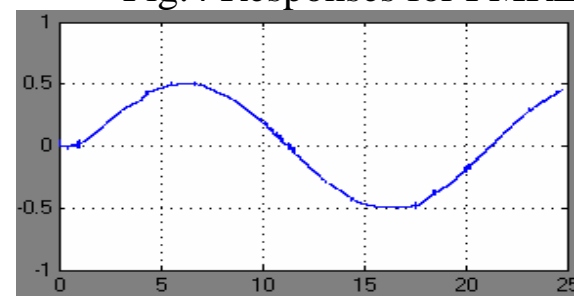

a) Speed response

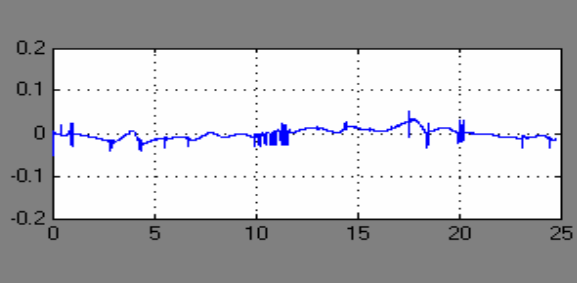

b) Tracking error

Fig.5 Responses for FMRLC (sinusoidal input sequence)

According to the environmental adaptability, a new study based on dynamic focusing fuzzy model reference learning control, under the control of the robot can adapt to the changing role of the parameters and the environment itself, and has a good balance of performance, high accuracy and robustness advantages. Control algorithms are simulated by MATLAB and Simulink, verify the correctness and validity of the design. 
Figure 6 is a result of the robot tracing a straight line of $y=x+5$, a) in the trajectory of the robot coordinate plane, b) shows the convergence of the state vector. The results show that the robot can accurately track lines, circles, and other trace curve, error convergence fast movement is very flexible, adaptable terrain.

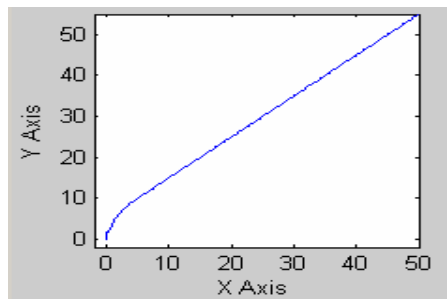

a) Tracking straight line $y=x+5$

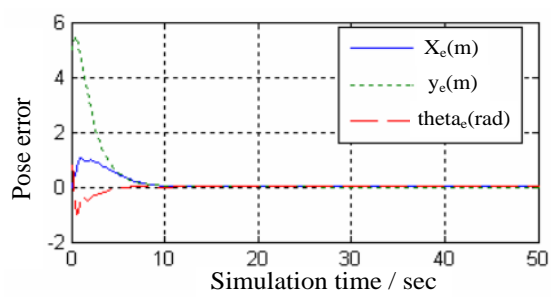

b). PE state vector changes Fig. 6 Line tracking of two-wheeled mobile robot

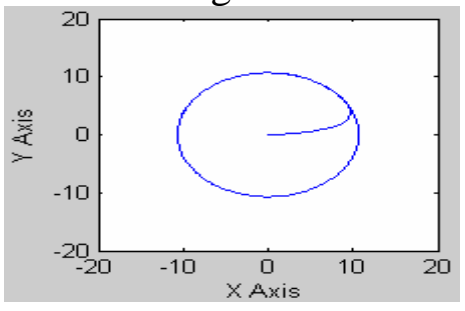

Fig.7Circle tracking

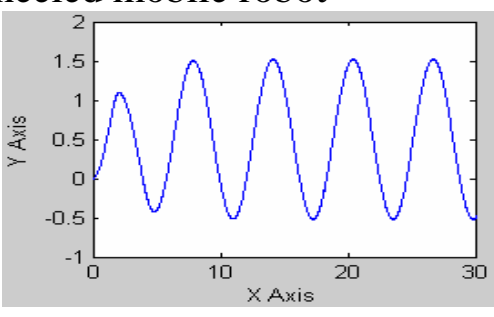

Fig.8Sine wave tracking of two-wheeled mobile robot

Figure 7 and Figure 8 shows the two self-balancing robot trajectory tracking more complex capabilities. The simulation demonstrates the validity of the method.

\section{Experimental study of the system}

In order to adjust the parameters and verify the validity of the Kalman filter is a Kalman filter, the Kalman filter filtering the test platform to complete the experiment: the sensor module is connected to a potentiometer variable knob, rotation sensor within a certain range, the inclination obtained by a potential meter true value, and storing the angle and the angular velocity measurements accelerometers and gyroscopes obtained. Noise covariance matrices Q and Rare obtained through several experiments $\mathrm{Q}=$ [0.05 0; 0 0.01]; $\mathrm{R}=$ [0.05]; experimental results are shown in Fig.9.

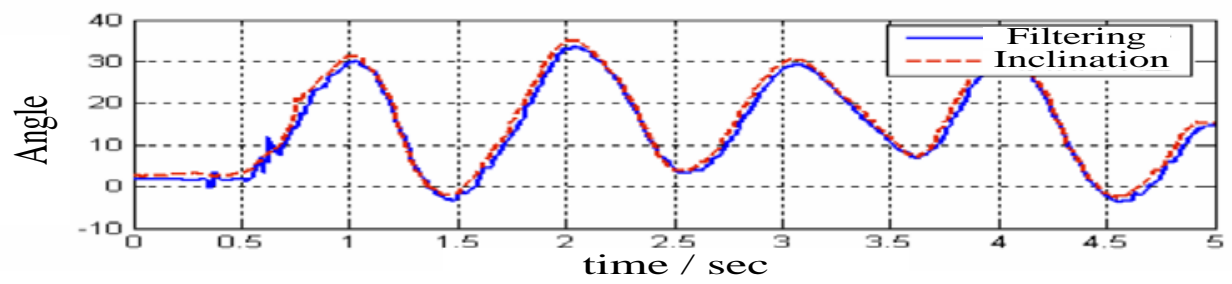

Fig.9 Comparison between estimated angle and true angle

Seen from Figure 9, the Kalman filter (up to about 3 degrees) and dip in the initial stages when the error is greater the larger the following reasons.

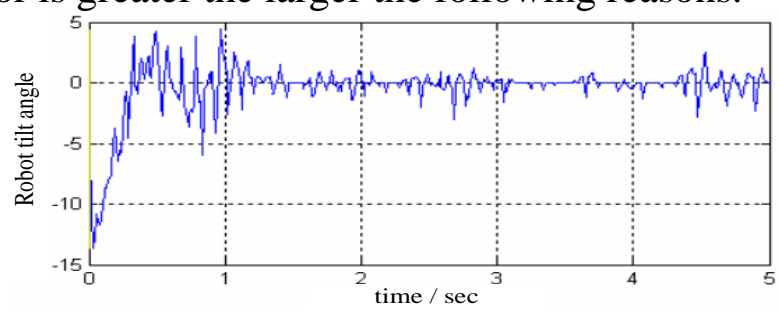

Fig.10 Balance experiment without load

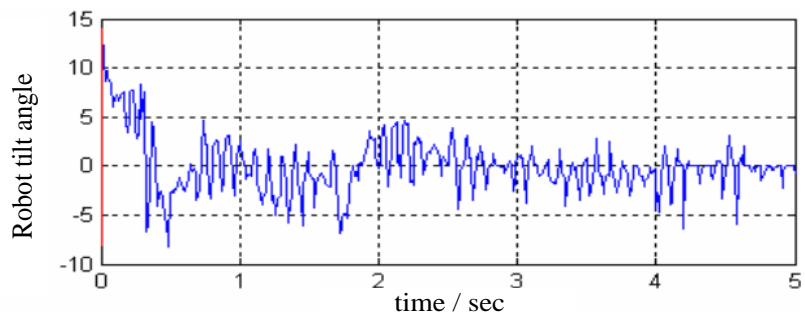

Fig.11 Balance experiment with load

When seen from Figure 10, the robot from the load, the initial angle is about 12 degrees; after 0.5 seconds tend to balance the vibration of the rapid state of the body in a second range of tilt is no longer a large oscillation, the fuzzy verification model Reference learning Controller has a strong balance. Figure 11 shows the robot loads the quality of $0.4 \mathrm{~kg}$, and because of the presence of the load, 
${ }^{[5-9]}$ the robot body weight increases, shifting the center of mass, the robot can achieve balance control through learning.

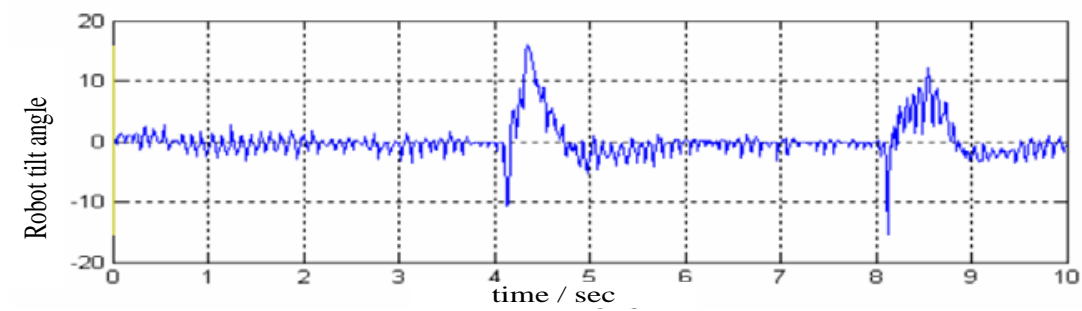

Fig.12 Robust experiment

Seen from Figure 12, the robot suddenly imposed by external forces under external force, within 0.6 seconds of you can quickly return to equilibrium, showed linear quadratic optimal tracking controller is real good, good for the inhibitory effect of external interference to verify the ability to suppress interference robustness.

\section{Summary}

In this paper, two-wheeled self-balancing robot as research object, first introduced the two-wheeled self-balancing robot test platform, and then for the sensor data fusion based on indirect Kalman filter, balance control, robustness and speed tracking experiments conducted research on mathematical model, sensor data processing, and speed controller, position controller design, carried out research. The experimental data have been analyzed experimental results verify the correctness and validity of theoretical research.

\section{References}

[1] Felix Grasser, Aldo Dario, Silvio Colom bi, Alfred C. Rufer. JOE: A Mobile, Inverted Pendulum. IEEE Transactions on Industrial Electronics, vol. 49, No.1, February 2012

[2] Giuseppe Oriolo, Alessandro De Luca, Marilena Vendittelli. WMR Control via Dynamic Feedback Linearization: Design, Implementation, and Experimental Validation. IEEE Transactions on Control Systems Technology, vol.10, No.6, November 2012

[3] H. K. Lam, T. H. Lee, F. H. F. Leung, P. K. S. Tam. Fuzzy Model Reference Control of Wheeled Mobile Robots. The 27th Annual Conference of the IEEE Industrial Electronics Society

[4] Hugh H. S. Liu, Grantham K. H. Pang. Accelerometer for Mobile Robot Positioning. IEEE Transactions on Industry Applications, Vol. No.3, May 2011

[5] Ashraf Enlarger. Prediction of Moving Objects in Dynamic Environments Using Kaman Filters. Proceedings of 2001 IEEE International Symposium on Computational Intelligence in Robotics and Automation July 29-August1, 2011

[6] Saticoy FURUKI, Shin 'chi YUTA, Pose Estimation of a Vehicle on Rough. Terrain by Using the Sun Direction. IEEE Conference on Multisensory Fusion and Integration for Intelligent Systems 2003

[7] Albert-Jan, Baer veldt, Robert Klan. A Low-cost Low-weight Attitude Estimation. System for an Autonomous Helicopter

[8] Ruth E. Mayagoitia, Amanda V. Nene, Peter H. Vesting. Accelerometer and rate gyroscope measurement of kinematics: an inexpensive alternative to optical motion analysis systems. Journal of Biomechanics 35(2002) p.537-542

[9] Dan Harmoniums. A low-cost micromechanical accelerometer with integrated solid-state sensor. Sensors and Actuators 84(2000) p.149-155 